\title{
Evaluación del Contenido de Licopeno en Pastas de Tomate Comerciales
}

\author{
Celeste Fernández, Alberto Pitre, María J. LLobregat y Yadrenia Rondón \\ Universidad de Carabobo, Facultad de Ingeniería, Escuela de Ingeniería Química, \\ Centro de Investigaciones Químicas, Av. Universidad, Bàrbula, Valencia-Venezuela. \\ (e-mail: cfernand@uc.edu.ve)
}

\begin{abstract}
Resumen
Se ha evaluado el contenido de licopeno y algunas propiedades fisicoquímicas y microbiológicas en cuatro pastas de tomates, tres nacionales y una importada. La metodología consistió en la extracción de los compuestos carotenoides presentes en las pastas de tomates con acetona. Los extractos carotenoides se separaron y purificaron por cromatografía de capa fina utilizando como solvente éter de petróleo ligero, y se prepararon soluciones estándares de licopeno. Usando espectrofotometría UV-visible, se determinaron las concentraciones de licopeno. Se determinó además, los sólidos solubles, acidez iónica, acidez titulable y cloruros totales, siguiendo los métodos recomendados por la comisión venezolana de normas industriales COVENIN. Se encontró que las pastas de tomate con mayor contenido de licopeno son la importada con $0.1305 \mathrm{ppm}$ y una nacional con $0.1271 \mathrm{ppm}$, lo que sugiere que el consumo de pasta nacional también brinda los beneficios aportados por el licopeno, siendo ésta un producto más barato (relación 1:7 en Venezuela). En relación a las propiedades fisicoquímicas, todas las pastas estudiadas satisfacen las especificaciones establecidas por las normas COVENIN.
\end{abstract}

Palabras claves: licopeno, pasta de tomate, cromatografía de capa fina, compuestos carotenoides

\section{Determination of the Lycopene Content in Different Commercial Tomato Pastes}

\begin{abstract}
Lycopene content and some physicochemical and microbiological properties of tomato pastes, three made in Venezuela and one imported, were determined. The methodology consisted of the extraction of the carotenoid compounds present in the tomato pastes with acetone. Then, the carotenoid extract was separated and purified by thin layer chromatography using light oil ether as solvent. Several standard solutions of Lycopene were prepared and the concentrations of licopene were determined using UV spectrophotometry. Also, soluble solids, ionic acidity, acidity by titration, and total chlorides were determined by using the methods recommended by the Venezuelan commission of industrial norms, COVENIN. The results showed that the imported tomato paste exhibits the highest Lycopene content with $0.1305 \mathrm{ppm}$, followed by one of the Venezuelan pastes with a content of $0.1271 \mathrm{ppm}$. These results indicate that the consumption of national tomato pastes also gives the benefits of the Lycopene. Whit regard to the physicochemical properties, the results are in agreement with the specifications established by the COVENIN norms.
\end{abstract}

Keywords: lycopene, tomato paste, thin layer chromatography, carotenoid compounds 


\section{INTRODUCCION}

Los carotenoides son compuestos solubles en lípidos, y son los encargados de dar color a los frutos y vegetales, entre los más importantes para el organismo se tienen los: $\beta$-carotenos, $\alpha$-carotenos, licopeno, criptoxantina, luteína y zeaxantina (Nguyen y Schwart, 1999). El licopeno es el pigmento responsable del color rojo que presentan los tomates, pomelos, sandías, pimentones, etc. El licopeno es un colorante con una estructura química de cadena abierta con once dobles enlaces conjugados, de estructura sencilla con una cadena alifática formada por cuarenta átomos de carbono, éste se absorbe mejor a través de las grasas y aceites por su liposolubilidad y se encuentra presente en el organismo humano tanto en la sangre como en tejidos (Perking-Veazie et al., 2001).

El estilo de vida actual obliga a las personas a consumir alimentos procesados que no tienen la mismas características nutrimentales que los frescos y el reto es elaborar productos fáciles de preparar y de alta calidad nutricional. Los carotenoides han cobrado gran importancia debido a que son antioxidantes que neutralizan los radicales libres que dañan a las células, de ellos, el licopeno posee propiedades antioxidantes mucho más potentes que el $\beta$-caroteno, y actúa protegiendo las células del estrés oxidativo producido por la acción de los radicales libres (Candelas et al., 2005), que es uno de los responsables de cáncer, enfermedades cardiovasculares y envejecimiento. Este carotenoide se encuentra en el tomate fresco pero también en los productos procesados, como el tomate deshidratado, pasta de tomate y en polvo (Clinton, 1998).

La degradación de los carotenoides se debe fundamentalmente a las reacciones de oxidación y se presentan generalmente durante el secado de frutas y vegetales, por ejemplo el licopeno, pigmento responsable de la coloración de los tomates, es muy estable en ese fruto, pero extraído y purificado es muy lábil (Meléndez et al., 2004). Desde el punto de vista nutricional, el problema más grande que tiene la ingesta de licopeno está en cómo consumirlo, el licopeno está más biodisponible cuando se somete a cocción (pasta de tomate), así es cuando mejor hace su efecto, cumpliendo sus propiedades como antioxidante y anticancerígeno (Méndez y Hernández, 2006). Estudios epidemiológicos sugieren que el consumo de licopeno tiene un efecto beneficioso sobre la salud humana, reduciendo la incidencia de las patologías cancerosas, otros estudios realizados por investigadores reveló que el consumo de licopeno redujo en un $45 \%$ la posibilidad de desarrollar cáncer en la próstata a diez raciones semanales de tomate o subproductos de éste. Otras investigaciones descubrieron que también reducen los niveles de colesterol en forma de lipoproteína de baja densidad que tienen incidencia en enfermedades cardiovasculares (Giovannucci et al., 2002).

Al momento de producir la pasta de tomate o cualquier otro producto derivado del tomate, se puede observar que la mayoría de estas carecen, en el etiquetado, de información nutricional, y las que tienen dicha información, no indican el nivel de licopeno o carotenoides presentes en ella. El conocimiento de los beneficios aportados por el licopeno, motivó la investigación aquí descrita, en la que se evaluaron pastas de tomate de tres diferentes marcas comerciales nacionales y una pasta de tomate comercial importada, permitiendo establecer una comparación entre las mismas con el fin de verificar los niveles de licopeno, y las propiedades fisicoquímicas y microbiológicas, siguiendo para ello, los métodos establecido por la Comisión Venezolana de Normas Industriales COVENIN, arrojando como resultado que la pasta de tomate importada es la que aporta el mayor contenido de licopeno.

\section{MATERIALES Y MÈTODOS}

\section{Materia Prima}

Se identificaron la existencia de diferentes marcas de pastas de tomate en centros de abastecimientos de víveres ubicados en el municipio Valencia del estado Carabobo, se solicitaron datos sobre el consumo de las diferentes marcas de pasta de tomate comerciales en los centros de acopio, se comparó el costo entre ellas verificando que estas sean accesibles al consumidor, con fecha de expiración de un año, y posteriormente se seleccionaron tres pastas de tomate nacionales , que etiquetamos como patas A, B, C y una pasta importada con etiqueta $D$. 


\section{Preparación de las muestras}

Una vez seleccionadas las pastas de tomates, se procedió a pesar $5 \mathrm{~g}$ de muestra, ésta se trituro en un mortero con $25 \mathrm{ml}$ de acetona, y se trató repetidas veces con el solvente hasta obtener los extractos incoloros, dichos extractos acètonicos se filtraron y se evaporaron hasta sequedad para diluirlos seguidamente en éter etílico en un embudo de separación con $25 \mathrm{ml}$ de $\mathrm{NaCL}$ al $10 \%$, luego se mezclaron agitando cuidadosamente. Posteriormente se decantaron y se separaron la fase acuosa y etérea, el lavado se realizó sucesivamente hasta que la fase acuosa se hiciera incolora, se deshidrató la fase etérea y se llevó a sequedad en un rotavapor durante una hora, la muestra finalmente seca se diluye en acetona con el objetivo de realizar la Cromatografía de Capa fina. Todo el proceso se llevó a cabo bajo luz reducida (Minguez, 1997).

\section{Extracción del licopeno}

Los pigmentos carotenoides fueron separados y purificados a través de la cromatografía de capa fina utilizando placas de silicagel $60 \mathrm{GF}_{254}$ de 20x20, como solvente de desarrollo se utilizó éter de petróleo ligero (Minguez, 1997). Las placas fueron activadas en la estufa a $120^{\circ} \mathrm{C}$ durante una hora antes de ser utilizadas, el extracto carotenoide se colocó sobre la placa en pequeñas proporciones utilizando la pipeta Pasteur, se llevaron a la cámara de desarrollo por espacio de (15 a 30) minutos, se extraen las placas, luego se secan para finalmente colocarlas bajo la luz ultravioleta, tanto el recorrido del solvente como de los pigmentos carotenoides son marcados y medidos para calcular la movilidad relativa (Rf) e identificarlos. El cálculo del Rf viene dado por la siguiente expresión.

$\mathrm{Rf}=\frac{\text { Drcomp }}{\text { Drsolv }}$

Donde Rf es la movilidad relativa, Drcomp representa la distancia recorrida por la mancha de un compuesto, Drsolv es la distancia recorrida por el solvente al momento de sacar la placa $(\mathrm{cm})$.

Se usa la Ecuación de Lambert-Beer al momento de determinar la absorbancia teóricamente, utilizando un estándar de concentraciones conocidas de licopeno.

$A=I \times E^{1 \%} \times C$

Donde A es la absorbancia $(\mathrm{Au})$ y I representa la longitud de la celda $(\mathrm{cm}), \mathrm{E}^{1 \%}$ es el coeficiente de extinción de una solución al 1\% en una celda de un $\mathrm{cm}((\mathrm{mlx} 100) /(\mathrm{gxcm}))$ cuyo valor es de 3450 para el licopeno en hexano y $\mathrm{C}$ es la concentración $(\mathrm{g} / \mathrm{L})$

\section{Preparación del patrón de licopeno}

Se utilizó como patrón licopeno sigma, $5 \mathrm{mg}$ de licopeno se diluyeron en $10 \mathrm{ml}$ de hexano, a partir de esta muestra se prepararon distintas diluciones sucesivas en una atmósfera confinada de nitrógeno, en un rango de 1 a 4 ppm, los extractos fueron evaluados mediante espectroscopía visible utilizando un Spectronic 20, a una longitud de onda de $450 \mathrm{~nm}$. La determinación del licopeno total se efectuó, mediante la siguiente curva de calibración $Y=0,2386 X-0.0718$.

\section{Cuantificación del contenido de licopeno presente en cada pasta de tomate comercial}

Una vez extraídos los pigmentos de las pastas de tomate comerciales, a un determinado peso de muestra, y concluido el desarrollo cromatográfico en capa fina, se raspó la capa de forma individual para cada uno de los componentes a una movilidad relativa Rf de 0.15 , se diluyó con hexano y se determinó la absorbancia a la longitud de onda de máxima absorción, se sustituye en la ecuación de Lambert-Beer y se determina las concentraciones de licopeno en las distintas pastas de tomate, la identificación del licopeno se basó en el tiempo de retención del estándar o patrón de licopeno. 


\section{Características fisicoquímicas y microbiológicas}

Con la finalidad de asegurar la calidad de las muestras, se evaluaron las siguientes características fisicoquímicas y microbiológicas de las pastas de tomate seleccionadas, en cuanto a la parte microbiológica; se realizaron los análisis correspondientes sobre métodos para recuento de bacterias aerobias en placas de petri establecidas en las normas COVENIN 0902(1987) y sobre alimentos comercialmente estériles COVENIN 2278(1985), mientras que para los análisis fisicoquímicos se les determinó los sólidos solubles, con el refractómetro ABBÈ mediante la metodología establecida en las normas COVENIN 924(1983) expresando los resultados como Brix, el contenido de sal (\%) mediante el método COVENIN 1193(1981), la acidez titulable, expresada en porcentaje de ácido acético establecida en la norma COVENIN 1151 (1977) y la acidez iónica determinada mediante un phmetro marca Orión y la norma COVENIN 1315(1979).

En cuanto a la evaluación de la estabilidad de la pasta comercial seleccionada, se realizó el proceso de extracción y cuantificación del licopeno a la pasta por duplicado, se tomaron pastas del mismo tipo; en fechas cuatro meses anteriores a las fechas de expiración indicadas en las etiquetas, para asegurar el estudio de degradación del licopeno en un rango de tiempo considerable, el estudio abarco un periodo de cuatro semanas (Meléndez et al., 2004).

\section{RESULTADOS Y DISCUSIÓN}

El criterio escogido para la selección de las pastas de tomate comercial nacionales resultó de la evaluación de los métodos convencionales, desde inventarios en los diferentes establecimientos que abarcan estratos socioeconómicos heterogéneos hasta la solicitud de datos estadísticos sobre el consumo de las diferentes marcas de pastas de tomate comerciales en los centros de acopio. Se decidió tomar tres marcas de pasta de tomate comercial con el mayor número de coincidencias en los diferentes centros de abastecimiento, con rangos de costos accesibles. La selección de la pasta de tomate comercial importada se realizó de la misma forma que las marcas nacionales. Las extracciones se hicieron por duplicado para cada marca de pasta de tomate comercial. A medida que se efectuaron las extracciones de los pigmentos carotenoides se notaron los cambios en la coloración de la pasta; puesto que se partió de una pasta color rojo intenso y después de triturar varias veces el producto con el solvente utilizado, se adquirieron soluciones con distintos matices al color de origen.

En relación a la separación de los distintos pigmentos carotenoides se efectuó el proceso conocido como cromatografía de capa fina (TLC). Al realizar la TLC para las distintas marcas de pastas de tomate, se elucidaron desarrollos de diferentes componentes en las placas en cuanto al recorrido del solvente y la distancia recorrida por la mancha de la pasta a estudiar, a pesar de que estas se realizaron bajo las mismas condiciones experimentales. Esto se debe a que existen diferentes pigmentos o impurezas en el desarrollo de las placas.

De las variedades estudiadas la pasta de tomate comercial que arrojó el mayor contenido de licopeno fue la importada $D$ y a nivel nacional fue la pasta $B$, esto se relaciona con los valores obtenidos de las distancias recorridas por el sistema eluente "éter de petróleo ligero" y las distancias recorridas por los diferentes componentes de cada pasta de tomate comercial representada en la cromatografía de capa fina, en la Tabla 1, se observa la movilidad relativa (Rf) promedio de cada marca de pasta de tomate, donde el licopeno presenta una movilidad relativa (Rf) teórico de 0.15 (Minguez, 1997); por lo que se recalcan los valores obtenidos cercanos a este. Los valores de Rf obtenidos por debajo de 0.15 se acercan a los valores de Rf reportados teóricamente (Minguez, 1997); para el Fitoflueno que es de 0.12. Es probable que el "Fitoflueno" formara parte de los carotenoides presentes en la marca comercial " $A$ " como se puede observar en la Tabla 1, los otros componentes separados en la cromatografía de capa fina presentaron valores de Rf que están por encima de los valores reportados en la bibliografía trabajando con éter de petróleo ligero como sistema eluente por lo que no se pudo identificar el tipo de carotenoide aplicando este método (Minguez, 1997). 
En la Tabla 2 se presentan los valores obtenidos de absorbancia a partir de la concentración patrón de licopeno. Una vez realizada la curva de calibración $Y=0,2386 X-0.0718$, se procede a tomar las absorbancia de las muestras de licopeno para cada pasta de tomate comercial, a un valor de longitud de onda $(\lambda)$ cercano a $450(\mathrm{~nm})$, valor teórico reportado en la bibliografía para el licopeno cuando se trabaja en el espectrofotómetro con hexano como solvente (Minguez, 1997) y calcular la concentración experimental. En el espectro de absorbancia se reportan tres picos uno a $450 \mathrm{~nm}$ y otros picos a longitudes de onda muy alejados 470 y $654 \mathrm{~nm}$, que indican la presencia de otros posibles compuestos.

Tabla 1: Movilidad relativa (Rf) en el extracto carotenoide de cada marca de pasta de tomate comercial.

\begin{tabular}{|c|c|c|c|c|}
\hline Marca & A & B & C & D \\
\hline \multirow{4}{*}{$\begin{array}{c}\text { Rf } \\
\text { (adim) }\end{array}$} & 0,06 & ------ & ------ & ------ \\
\cline { 2 - 5 } & 0,12 & ----- & 0,10 & 0,10 \\
\cline { 2 - 5 } & 0,14 & 0,15 & 0,15 & 0,15 \\
\cline { 2 - 5 } & 0,43 & 0,41 & 0,39 & 0,34 \\
\cline { 2 - 5 } & ---------- & 0,42 & 0,38 \\
\hline
\end{tabular}

Tabla 2: Absorbancia a diferentes concentraciones de licopeno (patrón)

\begin{tabular}{cc}
\hline $\begin{array}{c}\text { Concentración } \\
\left(\mathrm{ppm} \pm{ }^{*} \mathrm{E}-5\right)\end{array}$ & $\begin{array}{c}\text { Absorbancia } \\
\left(\mathrm{Au} \pm 5^{\star} \mathrm{E}-5\right)\end{array}$ \\
\hline 1 & 0.12688 \\
2 & 0.46976 \\
3 & 0.63454 \\
4 & 0.86713 \\
\hline
\end{tabular}

En la tabla 3, se presentan las concentraciones de licopeno obtenidas a partir de las absorbancia de las diferentes pastas de tomate comerciales, se aprecia una amplia variabilidad con respecto al mercado nacional, siendo la pasta de tomate de marca comercial B la que presenta el mayor nivel de concentración de licopeno, seguido de la marca comercial C" y finalmente la marca comercial A. Esto indica que el costo de la pasta de tomate comercial no está asociado a los niveles de concentración de licopeno presentes en las mismas, siendo el orden de costo de mayor a menor el siguiente: la marca A, B, C. Por lo que se puede aseverar que el consumo de pasta de tomate nacional también brinda los beneficios aportados por el licopeno. Además el costo de la pasta de tomate comercial importada $\mathrm{D}$ es aproximadamente 7 veces mayor al valor de la pasta de tomate comercial nacional, por lo que deja de ser atractiva y rentable para el consumidor. Los resultados de la caracterización fisicoquímica aplicada a las diferentes muestras en estudio, fueron llevados a cabo a una temperatura de $27^{\circ} \mathrm{C}$ y una presión de 1 atmósfera. En la tabla 4 , la acidez de las pastas de tomate fue evaluada por titulación con hidróxido de sodio y expresada como porcentaje de ácido acético.

Al realizar una comparación de estos resultados con los límites establecidos en la norma COVENIN 1151(1977), se tiene que los valores de las pastas nacionales: C, B y la importada D están dentro del rango de estudio, mientras que la pasta de tomate $A$ se destaca por poseer un índice de acidez mayor al valor máximo establecido en la norma lo cual se relaciona con el menor de $\mathrm{pH}$ obtenido. Con respecto al estudio de la acidez iónica se obtuvieron los siguientes resultados, ver tabla 5.

Los valores de $\mathrm{pH}$ obtenidos presentan un importante grado de variación entre las diferentes pastas de tomate en estudio, pero todos dentro del rango de 3.86 y 4.16 . Esta característica está proporcionalmente afectada por el índice de acidez, lo que implica que todo factor que vincule a la acidez titulable afectará directamente al $\mathrm{pH}$. Con respecto a los valores de sólidos solubles se tiene que existe una correlación prácticamente lineal entre el contenido de materia seca y los sólidos solubles. Los sólidos solubles pueden aumentar debido a la degradación de los polímeros de fructanos en moléculas de azúcares simples, o a la mayor pérdida de agua en la composición general del fruto maduro. La cantidad de azúcares, ácidos, constituyentes minerales en cenizas, vitamina $C$, entre otros, son los principales elementos cuando se hace referencia a los sólidos solubles presentes en la pasta de tomate. Los valores considerados en la norma COVENIN 924(1982) como vigentes son los señalados en la Tabla 6, estos permiten concluir que el contenido nutricional en cuanto a vitamina $C$ y proteínas están muy bien representados tanto en la pasta seleccionada B como en la $\mathrm{C}$. 
Tabla 3: Concentración de licopeno en las diferentes marcas de pastas de tomate comerciales

\begin{tabular}{ccc}
\hline $\begin{array}{c}\text { Marcas de } \\
\text { pastas de } \\
\text { tomates }\end{array}$ & $\begin{array}{c}\text { Absorbancia } \\
\left(\mathrm{Au} \pm 5^{\star} \mathrm{E}-5\right)\end{array}$ & $\begin{array}{c}\text { Concentración } \\
\left(\mathrm{ppm} \pm{ }^{*} \mathrm{E}-5\right)\end{array}$ \\
\hline $\mathrm{B}$ & 0.02860 & 0.12710 \\
$\mathrm{C}$ & 0.00740 & 0.09144 \\
$\mathrm{~A}$ & 0.00323 & 0.08655 \\
$\mathrm{D}$ & 0.03220 & 0.13051 \\
\hline
\end{tabular}

Tabla 5: Resultados de la variación del pH en la pasta de tomate a temperatura ambiente

\begin{tabular}{cc|c|c}
\hline \multicolumn{2}{c|}{$\begin{array}{c}\text { Acidez iónica } \\
(\mathrm{pH} \pm 0.01) \\
\text { adim }\end{array}$} & \multicolumn{2}{c}{$\begin{array}{c}\text { Límites fisicoquímicos de } \\
\mathrm{pH}(\text { adim })\end{array}$} \\
\hline $\mathrm{B}$ & 4.01 & & mín. \\
$\mathrm{C}$ & 4.16 & & \\
$\mathrm{D}$ & 4.09 & --- & 4.2 \\
$\mathrm{~A}$ & 3.86 & & \\
\hline
\end{tabular}

Tabla 4: Determinación del índice de acidez

\begin{tabular}{cc|cc}
\hline \multirow{2}{*}{$\begin{array}{c}\text { Acidez titulable } \\
(\mathrm{IA} \pm 0.01) \%\end{array}$} & \multicolumn{2}{|c}{$\begin{array}{c}\text { Límites fisicoquímicos } \\
\text { de la acidez titulable } \\
(\% \mathrm{p} / \mathrm{p})\end{array}$} \\
\hline $\mathrm{B}$ & 1.22 & & \\
Mín. & Máx. \\
$\mathrm{C}$ & 0.78 & & \\
$\mathrm{D}$ & 0.99 & 0.58 & 1.24 \\
$\mathrm{~A}$ & 1.52 & & \\
\hline
\end{tabular}

Tabla 6: Resultados de la variación de los sólidos solubles en las pastas de tomates

\begin{tabular}{|c|c|c|c|}
\hline \multirow{2}{*}{\multicolumn{2}{|c|}{$\begin{array}{l}\text { Sólidos solubles } \\
(S S \pm 0.1)^{\circ} \text { Brix }\end{array}$}} & \multicolumn{2}{|c|}{$\begin{array}{c}\text { Límites de los sólidos } \\
\left.\text { solubles ( }{ }^{\circ} \text { Brix }\right)\end{array}$} \\
\hline & & Mín. & Máx. \\
\hline B & 9.6 & \multirow{4}{*}{8} & \multirow{4}{*}{24} \\
\hline C & 8.3 & & \\
\hline D & 3.0 & & \\
\hline A & 2.6 & & \\
\hline
\end{tabular}

En la tabla 7, el porcentaje de sales ó cloruros totales es la suma de las concentraciones de todos los sólidos disueltos en la solución formada por agua y la pasta de tomate, donde la mayoría de las sales disueltas son compuestos inorgánicos, como sulfatos $\left(\mathrm{SO}_{4}{ }^{\circ}\right)$, cloruros $\left(\mathrm{Cl}^{-}\right)$, carbonatos $\left(\mathrm{CO}_{3}{ }^{\circ)}\right.$, bicarbonatos $\left(\mathrm{HCO}_{3}{ }^{-}\right)$, de $\mathrm{Ca}, \mathrm{Mg}$ y $\mathrm{Na}$. Ocasionalmente, pueden estar presentes en exceso y su precisión es determinante en el sabor del producto en estudio, al observar estos resultados se puede notar que la pasta de tomate importada no refleja valores en el rango de estudio, esto se debe a que la concentración de los carbonatos presentes en la manufactura de la pasta son de valores muy altos con respecto a las nacionales, por lo cual el rango de estudio según la norma debería ampliarse, y así poder reportar un valor considerable.

En cuanto a la esterilidad comercial la norma utilizada para su determinación es COVENIN 2278(1985), en nuestro caso la pasta de tomate libre de microorganismos significativos desde el punto de vista de salud pública, pero capaz de reproducirse en el alimento bajo condiciones normales, no refrigeradas, almacenamiento y distribución del producto. Este procedimiento se realiza para comprobar que los recipientes herméticamente cerrados, cumplen con los requisitos de control de la actividad metabólica de los microorganismos, como también su supervivencia. En el caso de la pasta de tomate con un intervalo $(0.90-0.60)$ de humedad, algunas bacterias, levaduras y hongos se multiplican, es importante la inhibición de esos microorganismos con el apoyo aportado por los análisis fisicoquímicos como $\mathrm{pH}$, temperatura, porcentaje de sólidos y los coliformes presentes. En la Tabla 8 se presentan los resultados de los análisis microbiológicos realizados en las diferentes pastas de tomates.

Observando los resultados señalados en la tabla 8, relativos a los análisis realizados al aplicar la metodología que se encuentra en las normas COVENIN 0902(1987) para el recuento de bacterias coliformes; y que manifiestan ausencia de microorganismos, se puede inferir que las pastas de tomate estudiadas son sometidas a un proceso de excelentes condiciones térmicas, en donde se controlaron tres variables importantes: 
Tabla 7: Porcentajes de cloruros totales presentes en las pastas de tomates comerciales

\begin{tabular}{cc|cc}
\hline \multicolumn{2}{c|}{$\begin{array}{c}\text { Cloruros totales } \\
(\mathrm{CT} \pm 0.01) \%\end{array}$} & \multicolumn{2}{|c}{$\begin{array}{c}\text { Valores límites de } \\
\text { los cloruros totales } \\
(\% \mathrm{p} / \mathrm{p})\end{array}$} \\
& \multicolumn{2}{c|}{ Mín. } & Máx. \\
\hline $\mathrm{B}$ & 2.54 & & \\
$\mathrm{C}$ & 1.37 & ---- & 3 \\
$\mathrm{D}$ & ---- & & \\
$\mathrm{A}$ & 1.52 & & \\
\hline
\end{tabular}

Tabla 8: Resultados de los análisis microbiológicos de las diferentes pastas de tomates comerciales

\begin{tabular}{cccc}
\hline Marcas & $\begin{array}{c}\text { Crecimiento } \\
\text { en caldo } \\
\text { nutritivo }\end{array}$ & $\begin{array}{c}\text { Coliformes } \\
\text { Totales } \\
\text { ( Ufc/ g) }\end{array}$ & $\begin{array}{c}\text { Coliforme } \\
\text { s Fecales } \\
\text { ( Ufc/ g) }\end{array}$ \\
\hline A & Negativo & $<1$ & $<1$ \\
B & Negativo & $<1$ & $<1$ \\
C & Negativo & $<1$ & $<1$ \\
D & Negativo & $<1$ & $<1$ \\
\hline
\end{tabular}

a) Si hubo presencia de bacterias esporuladas termofílicas, la temperatura normal de almacenamiento se encontró por debajo del rango termófilo. b) Control del rango de alta acidez, para que los microorganismos que no toleran la acidez, estén presentes pero incapacitados para crecer en el medio ácido. c) Utilización de un proceso combinado de calor-actividad de agua, al momento de prevenir germinación y deterioro de los mesófilos, termófilos esporulados (Hurtado, 1987).

La estabilidad del licopeno se evaluó en términos de la diferencia de su concentración expresada en ppm. En la tabla 9, se reporta el estudio realizado en un periodo de cuatro semanas de la concentración de licopeno para los cuatro extractos obtenidos de la pasta de tomate seleccionada $B$, la muestra tomada en la primera semana de estudio presento el más alto nivel de licopeno con respecto a las muestras tomadas posteriormente, esto se debe a las condiciones a que es sometida el producto como, actividad del agua, temperatura, luz, isomerización y oxidación (Minguez, 1997; Nguyen y Schwartz, 1999; Angelova y Warthesen, 2000a).

Tabla 9: Concentración de licopeno pasta de tomate comercial B

\begin{tabular}{|c|c|}
\hline $\begin{array}{c}\text { Concentración } \\
\left(\mathrm{ppm} \pm{ }^{*} \mathrm{E}-5\right)\end{array}$ & Tiempo(semanas) \\
\hline 0,12710 & 1 \\
0,11790 & 2 \\
0,10590 & 3 \\
0,09350 & 4 \\
\hline
\end{tabular}

\section{CONCLUSIONES}

Los análisis provenientes de la identificación de pigmentos determinados en extractos carotenoides, de las distintas pastas de tomates, arrojaron el licopeno con una movilidad relativa $\mathrm{Rf}=0.15$, y el fitoflueno con una movilidad relativa $\mathrm{Rf}=0.12$. De las pastas de tomates comerciales en estudio, las marca D (importada), y la marca B (nacional) reportaron los más altos niveles de concentración de licopeno

Los resultados obtenidos en el estudio de acidez en las distintas pastas de tomates, indicaron que la marca A posee un valor por encima del rango señalado en la norma, no siendo acta para el consumo humano, puesto que su acidez es alta. El estudio microbiológico realizado para comprobar la hermeticidad de los envases, arrojó que cumplen con los requisitos de control de actividad metabólica de los microorganismos.

La evidente degradación del licopeno, al parecer, indica que existen factores fisicoquímicos que alteran la muestra. 


\section{AGRADECIMIENTOS}

Los autores expresan su agradecimiento a la profesora Nancy Salinas de la Facultad de Ciencias y Tecnología de la Universidad de Carabobo (FACYT) y al Consejo de Desarrollo Científico y Humanístico CDCH-UC por el financiamiento otorgado al proyecto de investigación № 1558-03.

\section{REFERENCIAS}

Angelova, T y Warthesen, J., Lycopene stability in tomato powders, Journal of Food Science, 65(1), 67-70 (2000a).

Candelas, M. G., Alanìs, M. G., Bautista, M., Del Río, F. y García, C., Contenido de licopeno en jugo de tomate secado por aspersión, Revista Mexicana de Ingeniería Química, Vol.4, 299-307 (2005).

Clinton, S. K., Lycopene. Chemistry, biology and implications for human health and dissuade, Nutr. Rev., 56(2); 35-51 (1998).

COVENIN 1315, Comisión Venezolana de Normas Industriales, Frutas y productos derivados, Determinación del pH acidez iónica, Ministerio de Fomento, Caracas, Venezuela (1979).

COVENIN 1151, Comisión Venezolana de Normas Industriales, Frutas y productos derivados, Determinación de la acidez titulable, Ministerio de Fomento, Caracas, Venezuela (1977).

COVENIN 924, Comisión Venezolana de Normas Industriales, Frutas y productos derivados, Determinación de sólidos solubles por refractometría, Ministerio de Fomento, Caracas, Venezuela (1982).

COVENIN 1193, Comisión Venezolana de Normas Industriales, "Frutas y productos derivados, Determinación de cloruros totales". Ministerio de Fomento, Caracas, Venezuela (1983).

COVENIN 2278, Comisión Venezolana de Normas Industriales, Alimentos comercialmente estériles, Prueba de esterilidad comercial, Ministerio de Fomento, Caracas, Venezuela (1985).

COVENIN 0902, Comisión Venezolana de Normas Industriales, Alimentos. Método para el recuento de placas aerobias en placas de petri, Ministerio de Fomento, Caracas, Venezuela (1987).

Giovannuccci, E., Rimm, E. B., Stampfer, M. J y Willett, W. C., A prospective study of tomato products, licopene and prostate cancer risk, Journals of the national cancer institute, Mar 6; 94(5); 391-398 (2002).

Hurtado, F., Procesamiento del tomate y pulpas de frutas para exportación, Ediciones CIEPE, Venezuela (1987).

Meléndez, A.J., Vicario, I.M y Heredia, F., Estabilidad de los pigmentos carotenoides en los alimentos, Archivos latinoamericanos de nutrición, (en línea), Vol. 54 (2), 2004 (fecha de consulta Noviembre 11, 2006; www.alanrevista.org).

Méndez, R. M y Hernández, U. H., Contenido de licopeno en productos Mexicanos elaborados con jitomate. IV Congreso Internacional de Ingeniería Bioquímica y XV Congreso Nacional de Ingeniería Bioquímica. Moreli, Michoacán, México (2006).

Mìnguez, I.M., Clorofilas y carotenoides en tecnología de los alimentos, Universidad de Sevilla, (1997).

Nguyen, M. y Schwart, S., Lycopene: chemical and biological properties en "Food Technology", Vol. № 58(2), pp, 38-44 (1999).

Perking-Veazie, P., Collins, J., Pair, D. y Roberts, W., Lycopene content differs among red-fleshed watermelon cultivars en “J. Sci. Food Agric.”, Vol. No81, pp 983-087 (2001).

Rangana, S., "Manual of Analysis of Fruit and Vegetable Product", Tata McGraw-Hill Plant Pigments, 77-80 (1976). 